\title{
Detecting $J A K 2$ mutations in myeloproliferative neoplasms: can you please all the people all of the time?
}

\author{
Stephen E Langabeer* \\ Cancer Molecular Diagnostics, St. James's Hospital, Dublin, Ireland
}

The well-known adage "You can please some of the people all of the time, you can please all of the people some of the time, but you cannot please all of the people all of the time" is often attributed to John Lydgate, an English poet and translator born in the $14^{\text {th }}$ century, and was supposedly later paraphrased in an address by Abraham Lincoln, the $16^{\text {th }}$ President of the United States of America. This phrase is readily applicable to cancer molecular diagnostics when considering the variables of assay sensitivity and specificity in the detection of somatically acquired, disease-specific mutations. A molecular diagnostic assay should not only aspire to detect all mutations intended but also at a clinically relevant level of detection. This challenge is pertinent for the molecular detection of myeloproliferative neoplasm (MPN)-associated JAK2 mutations.

By far the most commonly acquired mutation in MPN is the p.V617F mutation (c.1849G $>$ T) located in JAK2 exon 14. It is present in more than $95 \%$ of patients with polycythemia vera and in more than half of those patients with essential thrombocythemia and primary myelofibrosis [1]. Identification of the JAK2 V617F and other so-called "driver" mutations in JAK2 exon 12, MPL exon 10 and CALR exon 9 is now an essential diagnostic criterion for MPN according to the World Health Organization classification of myeloid neoplasms [2]. Several methodologies exist to detect the JAK2 V617F mutation and include allele-specific PCR, quantitative PCR (qPCR), melting curve analysis, high resolution melt analysis, restriction enzyme digestion, direct sequencing, pyrosequencing, denaturing HPLC, digital droplet PCR and next-generation sequencing (NGS) [3]. Each of these techniques has its own sensitivity and specificity with adoption of the required technique largely dependent of the clinical requirement with qPCR most widely utilised to date due to low cost and excellent sensitivity [4]. Diagnostic guidelines have indicated a sensitivity of detection of approximately $2 \%$ mutant alleles for routine diagnostic purposes $[3,5,6]$ although it is widely acknowledged that $J A K 2 \mathrm{~V} 617 \mathrm{~F}$ allele burdens less than this can result in an MPN phenotype [7]. Importantly, several other JAK2 mutations have been described (in exon 14 and throughout the JAK2 gene) in both sporadic and familial MPN, albeit at a low incidence, and it is such cases that may be underdiagnosed to lack of specificity of assays that only target the JAK2 V617F [8,9].

The issue of sensitivity and specificity in the detection of JAK2 mutations has recently been comprehensively addressed by Maslah, et al. [10]. Firstly in a head to head comparison, this group observed excellent concordance between their JAK2 V617F qPCR and NGS approaches but only above the detection limit of their NGS assay which was $2 \%$ mutant alleles. However, due to the higher limit of detection of NGS, 25/473 (5.3\%) samples were found to be discordant between the two techniques with the majority of these discrepancies were due to the failure of NGS to detect low JAK2 V617F allele burdens less than $1 \%$. Conversely, qPCR failed to detect the alternative JAK2 exon 14 mutations of D620E, V615L and a different V617F resulting from a two nucleotide substitution. Secondly, this group used their NGS approach (all coding JAK2 exons) to screen a large cohort of patients with suspected MPN and discovered 28 variants in 52/473 (11.0\%) samples not including the V617F. Several of these variants were known single nucleotide polymorphisms (SNPs) or variants of unknown significance possessing allele burdens of approximately $50 \%$ most likely representing SNPs. Impressively, further somatic mutations $(\mathrm{D} 751 \mathrm{H}$, G281S, H345L, T817del) and variants previously described as germ line (E846D, N1108S, R1063H) were detected in 28/427 (6.6\%) patients [10].

Maslah, et al. eloquently demonstrate the value of both qPCR and NGS approaches for the detection of the JAK2 V617F and alternative $J A K 2$ mutations respectively. Clearly the advantage of $\mathrm{qPCR}$ is sensitivity at diagnosis and the capacity to quantitate low levels of measurable residual disease, especially relevant in the post-allogeneic stem cell transplant setting [11-13]. Despite this sensitivity, it is acknowledged that other MPN-associated JAK2 mutations can occur and are present in exon 14 and throughout the coding region and these mutations result in false-negative qPCR results $[14,15]$. However, frontline sequencing the entire coding region of JAK2 (and MPL) by NGS in all patients presenting with a thrombocytosis, an erythrocytosis, or any other MPN-related feature is probably unfeasible and unwarranted in the majority of diagnostic laboratories. Improvements are and will be made in the sensitivity of NGS approaches for detecting the JAK2 $\mathrm{V} 617 \mathrm{~F}$ at lower allele burdens suggesting the future possibility of this approach for tracking measurable residual disease [16, 17]. Current knowledge therefore mandates NGS of JAK2 (in clinically relevant cases) following JAK2 V617F qPCR for an optimal diagnosis.

\section{Competing interests}

The author declares no competing interests.

\section{References}

1. Helbig G (2018) Classical Philadelphia-negative myeloproliferative neoplasms: focus on mutations and JAK2 inhibitors. Med Oncol 35: 119. [Crossref]

${ }^{*}$ Correspondence to: Stephen E Langabeer, Cancer Molecular Diagnostics, Trinity Translational Medicine Institute, St. James's Hospital, Dublin, D08 W9RT , Ireland, E-mail: slangabeer@stjames.ie

Key words: myeloproliferative neoplasms, molecular diagnostics, JAK2 mutations, next-generation sequencing

Received: September 30, 2019; Accepted: October 29, 2019; Published: November 01, 2019 
2. Arber DA, Orazi A, Hasserjian R, Thiele J, Borowitz MJ, et al. (2016) The 2016 revision to the World Health Organization classification of myeloid neoplasms and acute leukemia. Blood 127: 2391-2405.

3. Bench AJ, White HE, Foroni L, Godfrey AL, Gerrard G, et al. (2013) Molecular diagnosis of the myeloproliferative neoplasms: UK guidelines for the detection of JAK2 V617F and other relevant mutations. Br J Haematol 160: 25-34. [Crossref]

4. Langabeer SE, Andrikovics H, Asp J, Bellosillo B, Carillo S, et al. (2015) Molecular diagnostics of myeloproliferative neoplasms. Eur J Haematol 95: 270-279.

5. Gong JZ, Cook JR, Greiner TC, Hedvat C, Hill CE, et al. (2013) Laboratory practice guidelines for detecting and reporting JAK2 and MPL mutations in myeloproliferative neoplasms: a report of the Association for Molecular Pathology. J Mol Diagn 15: 733-744.

6. Guglielmelli P, Pietra D, Pane F, Pancrazzi A, Cazzola M, et al. (2017) Recommendations for molecular testing in classical Ph1-neg myeloproliferative disorders: a consensus project of the Italian Society of Hematology. Leuk Res 28: 63-72.

7. Lippert E, Mansier O, Migeon M, Denys B, Nilsson A, et al. (2014) Clinical and biological characterization of patients with low (0.1-2\%) JAK2 V617F allele burden at diagnosis. Haematologica 99: e98-e101.

8. Ma W, Kantarjian H, Zhang X, Yeh CH, Zhang ZJ, et al. (2009) Mutation profile of JAK2 transcripts in patients with chronic myeloproliferative neoplasias. J Mol Diagn 11: 49-53.

9. Langabeer SE (2014) JAK2 mutations to the fore in hereditary thrombocythemia JAKSTAT 3: e957618. [Crossref]

10. Maslah N, Verger E, Schlageter MH, Miclea JM, Kiladjian JJ, et al. (2019) Nextgeneration sequencing for JAK2 mutation testing: advantages and pitfalls. Ann Hematol 98: 111-118.
11. Alchalby H, Badbaran A, Zabelina T, Kobbe G, Hahn J, et al. (2010) Impact of the JAK2 V617F status, allele burden, and clearance after allogeneic stem cell transplantation for myelofibrosis. Blood 116: 3572-3581.

12. Jovanovic JV, Ivey A, Vannucchi AM, Lippert E, Oppliger-Leibundgut E, et al (2013) Establishing optimal quantitative-polymerase chain reaction assays for routine diagnosis and tracking of minimal residual disease in JAK2 V61f-associated myeloproliferative neoplasms: a joint European LeukemiaNet/MPN\&MPNr-EuroNet (COST action BM0902) study. Leukemia 27: 2032-2039.

13. Haslam K, Molloy KM, Conneally E, Langabeer SE (2014) Evaluation of a JAK2 V617F quantitative PCR to monitor residual disease post-allogeneic hematopoietic stem cell transplantation for myeloproliferative neoplasms. Clin Chem Lab Med 52: e29-e31.

14. Warshawsky I, Mularo F, Hren C, Jakubowski M (2010) Failure of the Ipsogen Mutascreen kit to detect the JAK2 $617 \mathrm{~V}>\mathrm{F}$ mutation in samples with additional rare exon 14 mutations: implications for clinical testing and report of a novel $618 \mathrm{C}>\mathrm{F}$ mutation in addition to $617 \mathrm{~V}>\mathrm{F}$. Blood 115 : 3175-3176.

15. Tiong IS1,2, Casolari DA1,3, Moore S4, et al. (2017) Apparent 'JAK2-negative' polycythaemia vera due to compound mutations in exon 14. Br J Haematol 178: 333336. [Crossref]

16. Abdelhamid E, Figeac M, Renneville A, Quief S, Villenet C, et al. (2013) Quantification of JAK2 V617F mutation by next-generation sequencing technology. Am J Hematol 88: 536-537.

17. Stasik S, Schuster C, Ortlepp C, Platzbecker U, Bornhäuser M, et al. (2018) An optimized next-generation sequencing approach for sensitive detection of single nucleotide variants. Biomol Detect Quantif 15: 6-12.

Copyright: $@ 2019$ Langabeer SE. This is an open-access article distributed under the terms of the Creative Commons Attribution License, which permits unrestricted use, distribution, and reproduction in any medium, provided the original author and source are credited. 